\title{
«Auch die Patienten profitieren»
}

Interview: Bruno Kesseli

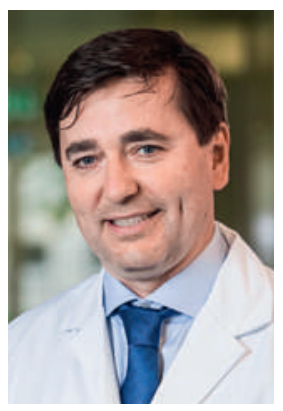

«Nachweislich erfolgreich»: Jens-Uwe Schaaf zum Projekt «Röntgenqualität in der Arztpraxis».
ALARA ist ein Akronym für «As Low As Reasonably Achievable». Das ALARA-Prinzip ist eine grundlegende Leitlinie des Strahlenschutzes.

Sinngemäss fordert das ALARA-Prinzip, beim Umgang mit ionisierenden Strahlen eine Strahlenbelastung von Menschen, Tieren und Material auch unterhalb von Grenzwerten so gering zu halten, wie dies mit vernünftigen Mitteln machbar ist.

** Medizinisch technische(r) Radiologieassistent(in)/ Radiologietechnologe/-in

bkesseli[at]emh.ch

Das im vorangegangenen Artikel vorgestellte Projekt «Röntgenqualität in der Arztpraxis» wurde von der swissradiology consulting initiiert, einem privaten Unternehmen, das im Bereich Qualitätssicherung in der Radiologie tätig ist. Im folgenden Kurzinterview beantwortet der ärztliche Leiter, Jens-Uwe Schaaf, einige Fragen zum Projekt.

Herr Schaaf, Ihre Firma positioniert sich als privater Dienstleister im Bereich Qualitätsmanagement Radiologie. Wie kam das Projekt «Röntgenqualität in der Arztpraxis» zustande?

Qualitativ hochwertige, standardisierte Röntgenuntersuchungen, die nach dem ALARA-Prinzip* angefertigt werden, sind neben der korrekten Indikation und Befundung die Grundlagen für eine gute radiologische Diagnostik. Das Projekt hat sich in einem Gespräch mit dem ärztlichen Leiter einer grossen Organisation von Gemeinschaftspraxen entwickelt, der in diesen eine Bewertung der Röntgenqualität nach den allgemein gültigen Radiologie-Standards, die in der Schweiz an Ausbildungsstätten für MPA und MTRA/RT** unterrichtet werden, durch meine Organisation wünschte. Das Projekt war mit seinen Ergebnissen in der Qualitätsverbesserung nachweislich erfolgreich. Es war deshalb nur konsequent, es auch anderen radiologisch tätigen Arztpraxen und Ärztenetzwerken vorzustellen.

Die Resonanz der involvierten Praxen scheint durchwegs positiv zu sein. Welcher Mehrwert ergibt sich aus dem Projekt für die angeschlossenen Ärztinnen und Ärzte und vor allem für deren Patientinnen und Patienten?

Die Radiologie-Experten der swissradiology consulting unterstützen die Kollegen und Kolleginnen in den Arztpraxen mit ihrem Wissen bei der Qualitätssicherung in der Röntgendiagnostik. Als Aussenstehende bewerten sie unvoreingenommen die Qualität der erbrachten Röntgenuntersuchungen und weisen auf Verbesserungspotentiale hin. Von dem Ergebnis dieser Zusammenarbeit können letztlich auch die Patientinnen und die Patienten profitieren.

Die SÄZ hat bei den Hausarztorganisationen nachgefragt, die dem Projekt durchaus positive Aspekte attestieren, aber auch - aus ihrer Sicht - unbefriedigende Punkte ausmachen. So wird bemängelt, dass es sich um eine rein technische Kontrolle ausgewählter Aufnahmen handelt. Ob die bei der Indikation zur Bildgebung gestellte Frage mit dem geprüften
Röntgenbild beantwortet werden konnte, werde nicht beurteilt. Diese Frage ist in der Praxis aber sicher entscheidend.

Das Projekt «Röntgenqualität in der Arztpraxis» ist modular aufgebaut. Neben dem "Grundmodul», bei dem die Bildqualität der Röntgenuntersuchung, unter Berücksichtigung der "technischen Indikation» und Einstelltechnik, bewertet wird, bieten wir ein erweitertes Modul an, das auch die ärztliche Indikation und den radiologischen Befund mit einbezieht. Wir möchten mit diesem differenzierten Angebot den ärztlichen Leitern der Arztpraxen die Möglichkeit geben, die Dienstleistung zu wählen, bei der sie glauben, Verbesserungen in ihrer Praxis erreichen zu können. Am 2.April 2015 habe ich diesen Projektaufbau persönlich dem Vorstand der Hausärzte Schweiz in Bern präsentiert.

Wenn ein privates Unternehmen in der Schweiz eine "flächendeckende" Ausweitung eines Projekts ins Auge fasst, entsteht damit der Eindruck, dass es ein Monopol anstrebt. Wie begegnen Sie solcher Skepsis? Eine flächendeckende» Ausweitung des Projektes zu verfolgen, bedeutet nicht automatisch eine Monopolstellung anzustreben. Die Aussage bezieht sich einzig auf die Projektziele und nicht auf meine Organisation. Da unsere Tätigkeit auf der Einhaltung definierter Standards in der Röntgendiagnostik beruht, können die Projektinhalte von anderen unabhängigen Organisationen übernommen und weitergeführt werden. Selbstverständlich sind wir auch gern zu Kooperationen bereit.

\section{Welche nächsten Schritte sind konkret geplant?}

Ein wichtiges Ziel für das Jahr 2016 ist es, die angesprochenen Hausarztorganisationen als Partner zu gewinnen und die derzeit noch bestehende Skepsis durch die Ergebnisse unserer Arbeit auszuräumen. Ich plane, radiologisch tätige Kollegen und Kolleginnen aus Arztpraxen mit in die Qualitätszirkel einzubeziehen, und werde die Zusammenarbeit mit anderen «Playern» im Gesundheitswesen, die sich für die Qualität von medizinischen Leistungen einsetzen, intensivieren. 